\title{
EFFICIENCY EVALUATION OF FUNCTIONING OF SOCIO-ECONOMIC SYSTEMS BASED ON PRODUCTION FUNCTIONS: NEW APPROACH ${ }^{1}$
}

\author{
Roman A. Zhukov \\ Financial University under the Government of the Russian Federation (Tula Branch), Tula, Russian Federation
}

\begin{abstract}
The aim of the article is to substantiate the possibility of using the performance indicator designed according to the author's methodology along with other indicators characterizing functioning of socio-economic systems. The performance indicator is defined as the ratio of normalized and standardized actual results to expected values which are calculated by the model of the relationship of effective signs and state and impact factors. The model is a production function, whose parameters can be defined by factor analysis of dependencies. To substantiate the presented indicator the author carries out a comparative analysis of its statistical dependence with the indicators of technical efficiency calculated using the stochastic boundary method. The paper considers 12 different variants which differ in the type of distributing the random component, which characterizes the inefficiency of the system; methods of calculating technical efficiency, as well as the presentation of data (pooled and panel data). As a basic model, the power multiplicative model in the form of Cobb-Douglas is used, which includes the volume of gross regional product, the cost of fixed assets, the average annual number of employees in economy and the cost of technological innovation taking into account inflation. The information base of the research is data on regions of the Central Federal district for 2007-2016. For the considered variants the relationships between the proposed performance indicator and other indicators considered were statistically significant, which gives reason to use it as a private indicator for analyzing functioning of socio-economic systems. The performance indicator can be used as a tool to analyze and compare functioning of socio-economic systems operating in different conditions.

Key words: mathematical model, performance indicator, technical efficiency, production function, stochastic frontier analysis, socio-economic system.
\end{abstract}

Citation. Zhukov R.A. Efficiency Evaluation of Functioning of Socio-Economic Systems Based on Production Functions: New Approach. Journal of Volgograd State University. Economics, 2019, vol. 21, no. 3, pp. 71-82. (in Russian). DOI: https://doi.org/10.15688/ek.jvolsu.2019.3.7

\section{ОЦЕНКА ЭФФЕКТИВНОСТИ ФУНКЦИОНИРОВАНИЯ СОЦИАЛЬНО-ЭКОНОМИЧЕСКИХ СИСТЕМ НА ОСНОВЕ ПРОИЗВОДСТВЕННЫХ ФУНКЦИЙ: НОВЫЙ ПОДХОД ${ }^{1}$}

\author{
Роман Александрович Жуков \\ Финансовый университет при Правительстве РФ (Тульский филиал), г. Тула, Российская Федерация
}

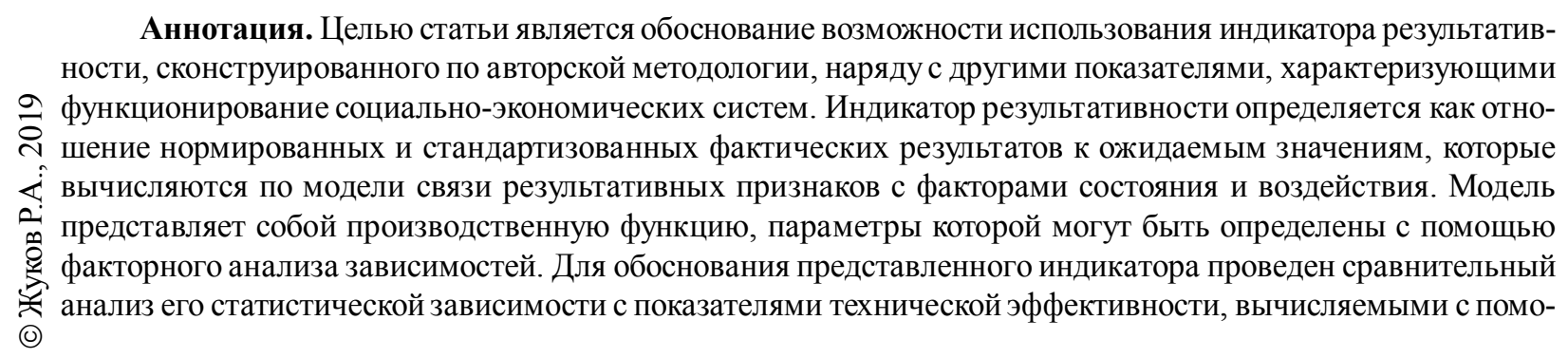


щью метода стохастической границы. Рассмотрено 12 различных вариантов, отличающихся видом распределения случайной составляющей, которая характеризует неэффективность функционирования системы; методами вычисления технической эффективности, а также представлением данных (объединенных и панельных). В качестве базовой модели использована степенная мультипликативная модель в форме Кобба - Дугласа, включающая объем валового регионального продукта, стоимость основных производственных фондов, среднегодовую численность занятых в экономике и затраты на технологические инновации с учетом инфляции. Информационной базой исследования стали данные по регионам Центрального федерального округа за 2007-2016 годы. Для рассмотренных вариантов связи между предложенным показателем результативности и другими рассмотренными индикаторами оказались статистически значимыми, что дает основание использовать его в качестве частного показателя для анализа функционирования социально-экономических систем. Показатель результативности может быть использован как инструмент анализа и сравнения функционирования социально-экономических систем, действующих в различных условиях.

Ключевые слова: математическая модель, показатель результативности, техническая эффективность, производственная функция, метод стохастической границы производственных возможностей, социальноэкономическая система.

Цитирование. Жуков Р. А. Оценка эффективности функционирования социально-экономических систем на основе производственных функций: новый подход // Вестник Волгоградского государственного университета. Экономика. - 2019. - Т. 21, № 3. - C. 71-82. - DOI: https://doi.org/10.15688/ek.jvolsu.2019.3.7

\section{Введение}

Современные подходы к оценке функционирования социально-экономических систем (СЭС) требуют от исследователей обоснования выбора инструментов и методов исследования для решения конкретной задачи. При этом возникающие трудности связаны с выбором индикаторов оценки и их экономическим содержанием, формированием требований к ним, корректным использованием методик построения таких индикаторов на базе экономико-математических моделей различной функциональной формы, определением области их применимости, а также с интерпретацией полученных результатов. Особый интерес представляет согласованность (соответствие) результатов оценок при использовании различных методов.

В статье рассматривается авторская методология формирования частных индикаторов оценки функционирования СЭС, которые сравниваются с показателями технической эффективности, вычисленными с использованием метода стохастической границы производственных возможностей (Stochastic Frontier Analysis (SFA)) для различного вида плотности распределения случайной составляющей, отвечающей за неэффективность производственного процесса.

Основной гипотезой исследования является утверждение о возможности использования сконструированных частных индикато- ров оценки взамен или в сочетании с показателями технической эффективности для изучения функционирования СЭС.

Объект исследования - регионы Центрального федерального округа (ЦФО), рассматриваемые как СЭС. Предмет исследования - согласованность результатов оценки функционирования СЭС, полученных с использованием различных методов.

Целью исследования является обоснование возможности использования частного индикатора оценки в качестве показателя, характеризующего результативность регионов СЭС по объему валового регионального продукта (ВРП).

\section{Современное состояние проблемы}

Проблематика оценки функционирования СЭС связана с понятиями результативности и эффективности, а также с построением соответствующих индикаторов оценки.

Традиционно результативность выражается в виде абсолютных значений показателей (например, объем ВРП, объем отгруженной продукции собственного производства по видам экономической деятельности и т. п.), которые ежегодно предоставляет Комитет государственной статистики Российской Федерации. Использование таких индикаторов при проведении сравнительного анализа функционирования СЭС, например, регионов - не совсем корректно, поскольку они находятся в 
различных условиях и имеют различные производственные возможности, которые и влияют на результат их деятельности. В этом аспекте под результативностью следует понимать показатель, учитывающий особенности функционирования СЭС с учетом существенных (значимых) факторов (условий), которые можно разделить на факторы состояния и воздействия.

Понятие эффективности функционирования СЭС перекликается с понятием результативности и подразумевает отношение результатов производства и факторов, обеспечивающих его заданное функционирование. Первыми публикациями, в которых был представлен термин «эффективность», являются работы Д. Риккардо, У. Петти и Ф. Кене, последние авторы отождествляли понятия результативности и эффективности [Айвазян и др., 2015, c. 8]. Так же как и ряд других исследователей в этой области [Котарбинский, 1975; Жуков, 2019], они рассматривали результативность (эффективность) как отношение фактического результата к ожидаемому значению.

Дальнейшее развитие методологии оценки связано с появлением концепции стохастической границы, основанной на теории X-эффективности [Leibenstein, 1966], введением термина «граница производственных возможностей», а также разделением «эффективности» на аллокативную, экономическую и техническую [Debreu, 1951; Farrel, 1957].

В 60-70-х годах прошлого столетия для оценки функционирования экономических систем разрабатывались параметрические и непараметрические методы. Наибольшей популярностью среди последних стал метод Data Envelopment Analysis (DEA), представленный группой исследователей - А. Чарнзом, В. Купером и Е. Родесом [Charnes et al., 1978]. В его основе лежит оценка эффективности посредством решения задачи линейного программирования. При этом не требуется четкая спецификация самой границы эффективности.

Наиболее известным из параметрических методов оценки стал метод стохастической границы производственных возможностей Stochastic Frontier Analysis (SFA), позволяющий построить стохастическую производственную функцию, которая впервые была представлена в 1977 году [Aigner et al., 1977]. Известно более 15 классических моделей, построенных с помощью SFA [Малахов и др., 2013]. Встречается ряд публикаций, где для поиска плотности распределения используется байесовский подход [Broeck et al., 1994; Kim et al., 2000].

Развитие информационных технологий позволило реализовать алгоритмы поиска эффективностей в виде отдельных программ (FRONTIER 4.1) и отдельных модулей в статистических (STATA) и математических (MathLab) [Makieta] пакетах, что существенно упростило проведение анализа функционирования СЭС для исследователей.

Ввиду большого разнообразия моделей в рамках SFA для оценки технической эффективности предлагаются различные алгоритмы выбора таких моделей [Айвазян и др., 2012]. При этом анализ показал, что их использование дает близкие в количественном выражении результаты, что приводит к выводу о целесообразности усложнения моделей в таких случаях. К тому же индикатор технической эффективности позволяет отличить производственную неэффективность от других неконтролируемых факторов, что ограничивает область его применимости. Поэтому в рамках данной статьи рассматривается возможность использования другого индикатора - показателя результативности для оценки функционирования СЭС.

\section{Методы и инструменты исследования}

Предположим, что результаты функционирования социально-экономической системы (СЭС) можно представить в виде:

$$
y_{i}=f\left(x_{i, i}\right)+\varepsilon_{i} .
$$

Здесь $\varepsilon_{i}-$ стохастическая случайная составляющая, которая может включать как результаты воздействия на функционирование СЭС - факторы, снижающие эффективность ее функционирования, так и факторы, которые не учтены в модели в явном виде и которые оказывают сбалансированное влияние на СЭС. $f\left(x_{i, j}\right)$ - функция, характеризующая ожидаемое (а в случае нормальности $\varepsilon_{i}$ - наиболее вероятное) значение результативного признака $\hat{y}_{i}$.

В случае если $y_{i}$ имеет смысл результатов производства для $i$-го объекта $-i$-й под- 
системы СЭС 1-го уровня, представляющей собой иерархическую структуру, то $\hat{y}_{i}+\varepsilon_{i}$ будет являться производственной функцией, определяющей максимально возможные объемы выпуска продукции при фиксированных объемах основных факторов производства $x_{i j}$ ( $j$ - индекс соответствующего фактора), которые можно разделить на факторы состояния и факторы воздействия.

В простейшем случае, когда $f\left(x_{i, j}\right)$ имеет линейный вид (или функция приведена к линейному виду), для оценки эффективности функционирования СЭС используют скорректированный метод наименьших квадратов (Corrected Ordinary Least Squares - COLS), добавляя к свободному члену модели максимальное значение из уровней ряда остатков $\varepsilon_{i}$.

Определение технической эффективности зависит от компонентного состава $\varepsilon_{i}$ и плотности вероятности, спецификации и функциональной формы $f\left(x_{i, j}\right)$, а также методов оценки технической эффективности. Однако техническая эффективность неполно отражает результаты функционирования СЭС, поскольку назначение этого индикатора - отличить производственную неэффективность от других неконтролируемых факторов, то есть без учета последних.

Поэтому в качестве индикатора оценки функционирования СЭС предлагается использовать не показатель технической эффективности, а индикатор результативности, характеризующий реальное функционирование СЭС с учетом существенных (значимых) и несущественных (не отраженных в модели) факторов, последние из которых могут иметь как положительное, так и отрицательное воздействие на результативный признак.

Поделив левую и правую части уравнения (1) на $f\left(x_{i, j}\right)$, получим:

$$
\frac{y_{i}}{f\left(x_{i, j}\right)}=\frac{y_{i}}{\hat{y}_{i}}=\frac{f\left(x_{i, j}\right)}{f\left(x_{i, j}\right)}+\frac{\varepsilon_{i}}{f\left(x_{i, j}\right)}=1+\frac{\varepsilon_{i}}{f\left(x_{i, j}\right)},
$$

где последнее слагаемое будет характеризовать как неэффективность функционирования СЭС, так и воздействие неучтенных в модели верхнего уровня $L_{1}$ факторов. Значение показателя, вычисленного по формуле (2) для $L_{1, i}$, меньшее единицы может служить основанием для более углубленного изучения ха- рактера влияния $x_{i, j}$ и $\eta_{i, k}$, последние из которых формируют $\varepsilon_{i}$.

При линейной функциональной форме выражения для вычисления ожидаемых значений $\hat{y}_{i}=f\left(x_{i, j}\right)$ показатель результативности и показатель эффективности, вычисленный с помощью метода COLS, согласуются с точностью до константы (свободного члена модели). При этом первый индикатор более нагляден, поскольку позволяет разделить изучаемые единицы совокупности, представляющие собой СЭС второго уровня, на более успешные (результативные), имеющие допустимую эффективность функционирования, и менее успешные, тогда как во втором случае граница разделения будет варьироваться в зависимости от значения $\varepsilon_{\max }$.

Определим частный индикатор результативности следующим образом:

$$
\xi_{k, i}(t)=\frac{y_{k, i}^{0}(t)}{\widehat{y}_{k, i}^{0}(t)}
$$

где $y_{k, i}^{0}(t), \hat{y}_{k, i}^{0}(t)$ - нормированные значения фактических и ожидаемых (нормативных, нормальных) результатов функционирования СЭС; $k$ - индекс (номер) СЭС; $i$ - индекс (номер) частного индикатора; $t$ - период оценки.

Нормированные значения определяются по формулам:

$$
\begin{aligned}
& y_{k, i}^{0}(t)=\frac{y_{k, i}^{*}-\min \left\{y_{k, i}^{*}, \widehat{y}_{k, i}^{*}\right\}}{\max \left\{y_{k, i}^{*}, \widehat{y}_{k, i}^{*}\right\}-\min \left\{y_{k, i}^{*}, \widehat{y}_{k, i}^{*}\right\}}, \\
& \hat{y}_{k, i}^{0}(t)=\frac{\widehat{y}_{k, i}^{*}-\min \left\{y_{k, i}^{*}, \widehat{y}_{k, i}^{*}\right\}}{\max \left\{y_{k, i}^{*}, \widehat{y}_{k, i}^{*}\right\}-\min \left\{y_{k, i}^{*}, \hat{y}_{k, i}^{*}\right\}},
\end{aligned}
$$

а стандартизованные значения $y_{k, i}^{*}(t), \widehat{y}_{k, i}^{*}(t)$ можно вычислить из соотношений:

$$
\begin{aligned}
& y_{k, i}^{*}(t)=\frac{y_{k, i}-M\left(y_{i}(t)\right)}{\sigma\left(y_{i}(t)\right)}, \\
& \hat{y}_{k, i}^{*}(t)=\frac{\hat{y}_{k, i}-M\left(\hat{y}_{i}(t)\right)}{\sigma\left(\hat{y}_{i}(t)\right)},
\end{aligned}
$$

где $y_{k, i}(t), \hat{y}_{k, i}(t)$ - значения фактических и ожидаемых результатов функционирования СЭС в абсолютных единицах измерения; $M\left(\hat{y}_{i}(t)\right), M\left(y_{i}(t)\right)$, $\sigma\left(\hat{y}_{i}(t)\right), \sigma\left(y_{i}(t)\right)$ - соответствующие им математические ожидания и стандартные отклонения. 
Ожидаемые результаты определяются функциональной зависимостью вида:

$$
\widehat{y}_{k, i}=f\left(x_{k, i, j}\right),
$$

входящей в состав соотношений (1) и (2) для $k$-й единицы совокупности.

Соотношения (6) и (7) обеспечивают безразмерность индикатора результативности, что позволяет сравнивать разнородные результаты функционирования СЭС, а (4) и (5) обеспечивают нормированность показателя, то есть числитель и знаменатель приводятся к интервалу от 0 до $1\left(y_{k, i}^{0}(t), \hat{y}_{k, i}^{0}(t) \in[0 ; 1]\right)$, снимая проблему масштаба для различных $i$-х индикаторов.

Для проведения сравнительного анализа оценки результативности функционирования СЭС представленного индикатора $\xi_{k, i}(t)$ с показателем технической эффективности в рамках метода SFA использовалось соотношение:

$$
y_{i}=f\left(x_{i, j}\right) \cdot \exp \left(-u_{i}\right) \cdot \exp \left(v_{i}\right)
$$

Здесь $u_{i}$ и $v_{i}-$ составляющие стохастической ошибки $\varepsilon_{i}$ в уравнениях (1) и (2), которые легко приводятся к мультипликативной форме. Выбор соответствующей формы определяется видом соотношения (8) для упрощения процедуры поиска параметров модели. В уравнении (9) для удобства индекс $k$ опущен, поскольку в рамках исследования модель строится для всех $k$ (объединенная (pooled) регрессия для панельных данных).

Техническая эффективность может быть определена посредством двух распространенных оценок JMLS (exp $\{-\mathrm{E}(\mathrm{u} \mid \varepsilon)\})$ [Jondrow et al., 1982] и $\mathrm{BC}(\mathrm{E}\{\exp (-\mathrm{u} \mid \varepsilon)\})$ [Battese et al., 1988].

Для проверки гипотезы о соответствии показателя результативности и технической эффективности был выбран критерий Стьюдента:

$$
t_{k r}=\sqrt{\frac{r^{2}}{1-r^{2}}(n-2)}>t_{\alpha, n-2}
$$

где $n$ - число наблюдений, $r$ - парный коэффициент корреляции между значениями показателя результативности и различными техническими эффективностями, $\alpha-$ уровень значимости.
Если условие (10) выполняется, то гипотезу $H_{0}$ о незначимости коэффициентов корреляции следует отвергнуть, то есть прийти к выводу, что между оценками (в данном случае $\xi_{k, i}(t)$, JMLS и BC) существует тесная статистическая связь, и первую из них можно использовать в качестве показателя результативности функционирования СЭС наряду с показателями технической эффективности.

\section{Базовая модель}

\section{и исходные данные исследования}

В качестве базовой модели была использована степенная мультипликативная модель, аналогичная модели Кобба - Дугласа [Cobb et al., 1928], в следующей форме [Жуков и др., 2018]:

$$
\widehat{y}_{10.1 / 2}=C_{0} \cdot x_{22.17 / 2}^{C_{2211}} \cdot x_{1 / 2}^{C_{1}} \cdot x_{2}^{C_{2}}
$$

где $\hat{y}_{10.1 / 2}$ - объем валового регионального продукта; $x_{22.17 / 2}$ - затраты на технологические инновации; $x_{1 / 2}-$ стоимость основных фондов по полной учетной стоимости на конец года; $x_{2}-$ среднегодовая численность занятых по видам экономической деятельности (всего); /2 - показывает, что значения показателей скорректированы на уровень инфляции и приведены к базисному периоду оценки (2007 г.); $C_{j}$ - соответствующие параметры модели, определяемые с помощью метода наименьших квадратов после проведения процедуры линеаризации.

Были проведены 4 вида оценок.

1. Показатель результативности $\xi_{k, i}$.

2. Техническая эффективность при условии, что плотность распределения $u_{i}$ имеет полунормальное распределение TE (halfnormal) $u_{i} \sim$ iid $N^{+}\left(0, \sigma_{u}^{2}\right)$ :

$$
f(u)=\frac{2}{\sqrt{2 \cdot \pi} \cdot \sigma_{u}} \cdot \exp \left(-\frac{u^{2}}{2 \cdot \sigma_{u}^{2}}\right) .
$$

3. Техническая эффективность при условии, что плотность распределения $u_{i}$ имеет экспоненциальное распределение ТЕ (exponential) $u_{i} \sim$ iid exp:

$$
f(u)=\frac{1}{\sigma_{u}} \cdot \exp \left(-\frac{u}{\sigma_{u}}\right) .
$$

4. Техническая эффективность при условии, что плотность распределения $u_{i}$ имеет 
усеченное распределение ТЕ (truncated normal): $u_{i} \sim$ iid $N^{+}\left(\mu, \sigma_{u}^{2}\right)$ :

$$
f(u)=\frac{2}{\sqrt{2 \cdot \pi} \cdot \sigma_{u}} \cdot \exp \left(-\frac{(u-\mu)^{2}}{2 \cdot \sigma_{u}^{2}}\right) .
$$

При этом выполняются условия:

$$
\varepsilon=-u+v, \quad \sigma_{\varepsilon}^{2}=\sigma_{u}^{2}+\sigma_{v}^{2}, \quad \lambda=\frac{\sigma_{u}}{\sigma_{v}} .
$$

В случаях (2)-(4) предполагается, что случайная величина подчиняется нормальному закону с плотностью распределения:

$$
f(v)=\frac{2}{\sqrt{2 \cdot \pi} \cdot \sigma_{v}} \cdot \exp \left(-\frac{v^{2}}{2 \cdot \sigma_{v}^{2}}\right) .
$$

Для оценки значимости использовался критерий Стьюдента.

Информационной базой исследования стали данные Федеральной службы государственной статистики за 2007-2016 гг. для регионов Центрального федерального округа без учета г. Москва. При этом строилась объединенная (pooled) регрессия по панельным данным.

Расчеты частного индикатора результативности проводились с использованием авторской экспертной системы (бета версия), а технические эффективности - с применением программного пакета STATA14. Данные вводились в логарифмированном виде.

\section{Результаты и обсуждение}

Были построены 4 модели в форме Кобба - Дугласа, спецификации которых представлены в таблицах 1-4.

Из таблиц видно, что все параметры моделей значимы, за исключением оценок $\sigma_{u}$ и $\lambda$. Это может свидетельствовать о том, что неэффективность производственного процесса незначима при изучении объема ВРП по панельным данным по совокупности регионов за 2007-2016 годы (столбец $\langle P>|t|\rangle)$. При таком масштабе исследования и предположении о независимости изучаемого процесса от фактора времени неэффективность с вероятностью 0,99 отсутствует.

Таблича 1

\section{Спецификация модели, полученной с помощью метода наименьших квадратов}

\begin{tabular}{|l|c|c|c|c|c|c|}
\hline Параметр & Значение & $\begin{array}{c}\text { Стандартная } \\
\text { ошибка }\end{array}$ & $t$-статистика & $P>|t|$ & \multicolumn{2}{|c|}{$\begin{array}{c}\text { \% дов ерительный } \\
\text { интервал }\end{array}$} \\
\hline$C_{1}$ & 0,5768899 & 0,0615344 & 9,38 & 0,000 & 0,4553989 & 0,6983808 \\
\hline$C_{2}$ & 0,5547885 & 0,0718472 & 7,72 & 0,000 & 0,4129363 & 0,6966406 \\
\hline$C_{22.17}$ & 0,0519689 & 0,0162104 & 3,21 & 0,002 & 0,0199637 & 0,083974 \\
\hline$C_{0}$ & 7,527867 & 0,4049884 & 18,59 & 0,000 & 6,728275 & 8,327459 \\
\hline
\end{tabular}

Примечание. Составлено автором. Число наблюдений -170 , коэффициент детерминации $\mathrm{R}^{2}=0,933$, критерий Фишера $\mathrm{F}=773,32$.

Спецификация модели, полученной

Таблица 2

с помощью метода максимального правдоподобия

в предположении о полунормальном виде распределения $\boldsymbol{u}$

\begin{tabular}{|l|c|c|c|c|c|c|}
\hline Параметр & Значение & $\begin{array}{c}\text { Стандартная } \\
\text { ошибка }\end{array}$ & $t$-статистика & $P>|t|$ & \multicolumn{2}{|c|}{$\begin{array}{c}\text { \% доверительный } \\
\text { интервал }\end{array}$} \\
\hline$C_{1}$ & 0,57689 & 0,0608059 & 9,49 & 0,000 & 0,4577125 & 0,6960674 \\
\hline$C_{2}$ & 0,5547884 & 0,0709966 & 7,81 & 0,000 & 0,4156376 & 0,6939392 \\
\hline$C_{22.17}$ & 0,0519688 & 0,0160185 & 3,24 & 0,001 & 0,0205731 & 0,0833645 \\
\hline$C_{0}$ & 7,529479 & 0,4139016 & 18,19 & 0,000 & 6,718247 & 8,340712 \\
\hline$\sigma_{u}$ & 0,0020496 & 0,1324483 & 0,02 & 0,988 & $2,02 \mathrm{e}-58$ & $2,08 \mathrm{e}+52$ \\
\hline$\sigma_{v}$ & 0,1738029 & 0,0094432 & 18,41 & 0,000 & 0,156246 & 0,1933325 \\
\hline$\lambda$ & 0,0117926 & 0,1333473 & 0,09 & 0,930 & $-0,2495633$ & 0,2731485 \\
\hline
\end{tabular}

Примечание. Составлено автором. Число наблюдений - 170 , критерий Вальда $\chi^{2}=2375,98$, значение функции правдоподобия MLE $=56,2471$. 
Спецификация модели, полученной

с помощью метода максимального правдоподобия

в предположении об экспоненциальном виде распределения $\boldsymbol{u}$

\begin{tabular}{|l|c|c|c|c|c|c|}
\hline Параметр & Значение & $\begin{array}{c}\text { Стандартная } \\
\text { ошибка }\end{array}$ & $t$-статистика & $P>|t|$ & \multicolumn{2}{|c|}{$\begin{array}{c}95 \text { \% доверительный } \\
\text { интервал }\end{array}$} \\
\hline$C_{1}$ & 0,5768961 & 0,0608053 & 9,49 & 0,000 & 0,4577198 & 0,6960724 \\
\hline$C_{2}$ & 0,5547842 & 0,0709956 & 7,81 & 0,000 & 0,4156353 & 0,6939331 \\
\hline$C_{22.17}$ & 0,0519662 & 0,0160184 & 3,24 & 0,001 & 0,0205708 & 0,0833617 \\
\hline$C_{0}$ & 7,532862 & 0,4075082 & 18,49 & 0,000 & 6,73416 & 8,331563 \\
\hline$\sigma_{u}$ & 0,0050245 & 0,0780079 & 0,06 & 0,949 & $3,06 \mathrm{e}-16$ & $8,25 \mathrm{e}+10$ \\
\hline$\sigma_{v}$ & 0,1737311 & 0,0096799 & 17,95 & 0,000 & 0,1557581 & 0,193778 \\
\hline$\lambda$ & 0,0289211 & 0,0807485 & 0,36 & 0,720 & $-0,129343$ & 0,1871852 \\
\hline
\end{tabular}

Примечание. Составлено автором. Число наблюдений - 170, критерий Вальда $\chi^{2}=2375,96$, значение функции правдоподобия MLE = 56,2466.

Таблица 4

Спецификация модели, полученной с помощью метода максимального правдоподобия в предположении об усеченном нормальном виде распределения $\boldsymbol{u}$

\begin{tabular}{|l|c|c|c|c|c|c|}
\hline Параметр & Значение & $\begin{array}{c}\text { Стандартная } \\
\text { ошибка }\end{array}$ & $t$-статистика & $P>|t|$ & \multicolumn{2}{|c|}{$\begin{array}{c}\text { \% доверительный } \\
\text { интервал }\end{array}$} \\
\hline$C_{1}$ & 0,5768899 & 0,0608062 & 9,49 & 0,000 & 0,4577119 & 0,6960679 \\
\hline$C_{2}$ & 0,5547884 & 0,070997 & 7,81 & 0,000 & 0,415637 & 0,6939399 \\
\hline$C_{22.17}$ & 0,0519688 & 0,0160186 & 3,24 & 0,001 & 0,020573 & 0,0833646 \\
\hline$C_{0}$ & 7,529108 & 0,4271288 & 17,63 & 0,000 & 6,691951 & 8,366265 \\
\hline$\mu$ & $-1,744782$ & 349,4737 & $-0,00$ & 0,996 & $-686,7007$ & 683,2112 \\
\hline$\sigma_{u}$ & 0,0465696 & 5,522218 & 0,01 & 0,993 & $5,4 \mathrm{e}-103$ & $4,01 \mathrm{e}+99$ \\
\hline$\sigma_{v}$ & 0,1738036 & 0,0094853 & 18,32 & 0,000 & 0,1561725 & 0,1934253 \\
\hline$\lambda$ & 0,2679439 & 5,522796 & 0,05 & 0,961 & $-10,55654$ & 11,09243 \\
\hline
\end{tabular}

Примечание. Составлено автором. Число наблюдений - 170 , критерий Вальда $\chi^{2}=2375,86$, значение функции правдоподобия MLE $=56,2471$.

В рамках исследования для оценки статистической связи между 4 индикаторами были вычислены коэффициенты парной корреляции. Результаты представлены в таблице 5.

Из таблицы 5 видно, что для half-normal и truncated normal распределений технические эффективности, вычисленные по JMLS и BC, практически идентичны (связь практически функциональная). Коэффициенты корреляции для ВС(е) отрицательны, что может быть объяснено неверной предпосылкой о виде распределения случайной величины, отвечающей за неэффективность.

Все связи между $\xi$ и JMLS и BC оценками статистически значимы, что может свидетельствовать о возможности использования представленного индикатора результативности в качестве показателя, характеризующего функционирование СЭС.

Поскольку в результате исследования оценки $\sigma_{u}$ оказались статистически незначимыми, то было принято решение рассмотреть исходные данные в пространственном и временном срезах (панельные данные). В этом случае регрессия может быть построена в двух вариантах: модель с фиксированными (fixed effects (FE)) и случайными (random effects (RE)) индивидуальными эффектами для выбранной совокупности объектов исследования [Wooldridge, 2002]. При этом для оценки результативности функционирования СЭС в соотношении (3) знаменатель будет содержать уже регрессионную модель, построенную по панельным данным. Для сравнения технические эффективности также необходимо рассчитать по панельным данным.

В рамках исследования были получены технические эффективности (JMLS и BC оценки) по панельным данным с использованием полунормальной, экспоненциальной и усеченной нормальной плотности распределения в $\mathrm{FE}$ и RE вариантах [Greene, 2005]. 
Коэффициенты корреляции между показателями функционирования СЭС

\begin{tabular}{|c|c|c|c|c|c|c|}
\hline Показатель & $\Xi$ & JMLS (h) & BC(h) & BC(e) & JMLS (t) & BC(t) \\
\hline$\xi$ & 1 & & & & & \\
\hline JMLS(h) & 0,920493 & & & & & \\
& $(30,350)$ & & & & & \\
& {$[0,000]$} & 1 & & & & \\
BC(h) & 0,920512 & 0,999997 & & & & \\
& $(30,354)$ & $(5237,809)$ & & & & \\
& {$[0,000]$} & {$[0,000]$} & 1 & & & \\
\hline BC(e) & $-0,33741$ & $-0,31335$ & $-0,31291$ & & & \\
& $(4,618)$ & $(4,251)$ & $(4,245)$ & & & \\
& {$[0,000]$} & {$[0,000]$} & {$[0,000]$} & 1 & & \\
\hline JMLS(t) & 0,919967 & 0,999982 & 0,999983 & $-0,31064$ & & \\
& $(30,238)$ & $(2169,681)$ & $(2230,664)$ & $(4,211)$ & & \\
& {$[0,000]$} & {$[0,000]$} & {$[0,000]$} & {$[0,000]$} & 1 & \\
\hline BC(t) & 0,919997 & 0,999981 & 0,999982 & $-0,31065$ & 0,999998 & \\
& $(30,244)$ & $(2095,528)$ & $(2175,162)$ & $(4,218)$ & $(6050,287)$ & \\
& {$[0,000]$} & {$[0,000]$} & {$[0,000]$} & {$[0,000]$} & {$[0,000]$} & \\
\hline
\end{tabular}

Примечание. Составлено автором. $\xi$-индикатор результативности; JMLS - оценка технической эффективностипо: [Jondrow et al., 1982], ВC- оценка технической эффективности по: [Battese et al., 1988], h-half-normal (полунормальное распределение), е- exponential (экспоненциальное распределение), $\mathrm{t}$ - truncated normal (усеченное распределение), (...)-t-статистика, [...]-p-value (вероятность принять гипотезу о незначимости коэффициента).

Оказалось, что модель с фиксированными эффектами неадекватна, поскольку коэффициент, стоящий перед переменной, характеризующей среднегодовую численность занятых в экономике, отрицателен. Поэтому в таблицах 6-9 приводятся спецификации только для RE моделей.

Из таблиц 6-9 видно, что параметры модели значимы на уровне 0,05 , за исключением $C_{22.17}$, который значим на уровне 0,188 и 0,136 для полунормального и усеченного нормального распределения соответственно. При этом в последнем случае математическое ожидание $\mu$ случайной величины $u$ оказалось незначимым, хотя значение симулятивной логарифмической функции правдоподобия мак- симально по сравнению с другими моделями. Значимыми оказались оценки $\sigma_{u}, \sigma_{v}$ и $\lambda$.

Результаты оценки парных коэффициентов корреляции между оценками для панельных данных представлены в таблице 10.

Таблица 10 демонстрирует практически функциональную связь между оценками для полунормального и усеченного нормального распределения. Связи между показателем результативности $\xi$ и показателями технической эффективности $J M L S(h), B C(h), B C(e), J M L S(t)$ и $B C(t)$ также статистически значимы, что дает основание представленный индикатор использовать в качестве оценки функционирования социально-экономических систем.

Таблища 6

Спецификация регрессионной модели, полученной

с помощью обобщенного метода наименьших квадратов по панельным данным

\begin{tabular}{|l|c|c|c|c|c|c|}
\hline Параметр & Значение & $\begin{array}{c}\text { Стандартная } \\
\text { ошибка }\end{array}$ & $t$-статистика & $P>|t|$ & \multicolumn{2}{|c|}{$\begin{array}{c}\text { \% доверительный } \\
\text { интервал }\end{array}$} \\
\hline$C_{1}$ & 0,8523109 & 0,0781135 & 10,91 & 0,000 & 0,6992112 & 1,005411 \\
\hline$C_{2}$ & 0,1714674 & 0,1139958 & 1,50 & 0,133 & $-0,0519601$ & 0,394895 \\
\hline$C_{22.17}$ & 0,0286432 & 0,0131453 & 2,18 & 0,029 & 0,0028789 & 0,0544074 \\
\hline$C_{0}$ & 6,590418 & 0,7139983 & 9,23 & 0,000 & 5,191007 & 7,989829 \\
\hline$\sigma_{u}$ & 0,15414952 & & & & & \\
\hline$\sigma_{v}$ & 0,09888642 & & & & & \\
\hline Rho & 0,0117926 & & & & & \\
\hline
\end{tabular}

Примечание. Составлено автором. Число наблюдений -170 , число групп -17 , критерий Вальда $\chi^{2}=345,05$, коэффициент детерминации $\mathrm{R}^{2}=0,9215, \mathrm{Rho}=\sigma_{\mathrm{u}}{ }^{2} /\left(\sigma_{\mathrm{u}}^{2}+\sigma_{\mathrm{v}}^{2}\right)$. 
P. А. Жуков. Оценка эффективности функционирования социально-экономических систем

Спецификация регрессионной модели, полученной с помощью лог-симулятивного метода максимального правдоподобия по панельным данным (плотность полунормального распределения $\boldsymbol{u}$ )

\begin{tabular}{|l|c|c|c|c|c|c|}
\hline Параметр & Значение & $\begin{array}{c}\text { Стандартная } \\
\text { ошибка }\end{array}$ & $t$-статистика & $P>|t|$ & \multicolumn{2}{|c|}{$\begin{array}{c}\text { \% доверительный } \\
\text { интервал }\end{array}$} \\
\hline$C_{1}$ & 0,8239741 & 0,067515 & 12,20 & 0,000 & 0,6916472 & 0,956301 \\
\hline$C_{2}$ & 0,2269606 & 0,0915235 & 2,48 & 0,013 & 0,0475778 & 0,4063434 \\
\hline$C_{22.17}$ & 0,0164105 & 0,0124644 & 1,32 & 0,188 & $-0,0080192$ & 0,0408402 \\
\hline$C_{0}$ & 6,824977 & 0,5102055 & 13,38 & 0,000 & 5,824993 & 7,824961 \\
\hline$\sigma_{u}$ & 0,154821 & 0,0231619 & 6,68 & 0,000 & 0,1154746 & 0,2075741 \\
\hline$\sigma_{v}$ & 0,0497874 & 0,0175045 & 2,84 & 0,004 & 0,0249948 & 0,0991719 \\
\hline$\lambda$ & 0,2857604 & 0,0721479 & 3,96 & 0,000 & 0,144353 & 0,4271677 \\
\hline
\end{tabular}

Примечание. Составлено автором. Число наблюдений - 170, число групп -17 , критерий Вальда $\chi^{2}=732,80$, значение симулятивной логарифмической функции правдоподобия $\mathrm{SML}=117,7488$.

Спецификация регрессионнй модели, полученной с помощью

Таблица 8 лог-симулятивного метода максимального правдоподобия по панельным данным (плотность экспоненциального распределения $\boldsymbol{u}$ )

\begin{tabular}{|l|c|c|c|c|c|c|}
\hline Параметр & Значение & $\begin{array}{c}\text { Стандартная } \\
\text { ошибка }\end{array}$ & $t$-статистика & $P>|t|$ & \multicolumn{2}{|c|}{$\begin{array}{c}\text { \% доверительный } \\
\text { интервал }\end{array}$} \\
\hline$C_{1}$ & 0,8472464 & 0,0665292 & 12,73 & 0,000 & 0,7168516 & 0,9776412 \\
\hline$C_{2}$ & 0,1864225 & 0,0947651 & 1,97 & 0,049 & 0,0006864 & 0,3721586 \\
\hline$C_{22.17}$ & 0,0307438 & 0,012616 & 2,44 & 0,015 & 0,0060169 & 0,0554707 \\
\hline$C_{0}$ & 6,572773 & 0,5875105 & 11,19 & 0,000 & 5,421274 & 7,724273 \\
\hline$\sigma_{u}$ & 0,0282658 & 0,0565213 & 0,50 & 0,617 & 0,0005613 & 1,423491 \\
\hline$\sigma_{v}$ & 0,0989143 & 0,0165518 & 5,98 & 0,000 & 0,0712564 & 0,1373076 \\
\hline$\lambda$ & 0,2857604 & 0,0721479 & 3,96 & 0,000 & 0,144353 & 0,4271677 \\
\hline
\end{tabular}

Примечание. Составлено автором. Число наблюдений -170 , число групп -17 , критерий Вальда $\chi^{2}=541,15$, значение симулятивной логарифмической функции правдоподобия $\mathrm{SML}=116,8585$.

Спецификация регрессионой модели, полученной с помощью

Таблича 9 лог-симулятивного метода максимального правдоподобия

по панельным данным (плотность усеченного нормального распределения $u$ )

\begin{tabular}{|l|c|c|c|c|c|c|}
\hline Параметр & Значение & $\begin{array}{c}\text { Стандартная } \\
\text { ошибка }\end{array}$ & $t$-статистика & $P>|t|$ & \multicolumn{2}{|c|}{$\begin{array}{c}\text { \% доверительный } \\
\text { интервал }\end{array}$} \\
\hline$C_{1}$ & 0,7810796 & 0,0685556 & 11,39 & 0,000 & 0,646713 & 0,9154461 \\
\hline$C_{2}$ & 0,3185162 & 0,1384036 & 2,30 & 0,021 & 0,0472501 & 0,5897824 \\
\hline$C_{22.17}$ & 0,0190589 & 0,0127724 & 1,49 & 0,136 & $-0,0059746$ & 0,0440925 \\
\hline$C_{0}$ & 6,761276 & & & & & \\
\hline$\mu$ & 0,0644644 & 0,099379 & 0,65 & 0,517 & $-0,1303149$ & 0,2592437 \\
\hline$\sigma_{u}$ & 0,1403576 & 0,0392022 & 3,58 & 0,000 & 0,0811883 & 0,2426492 \\
\hline$\sigma_{v}$ & 0,0436785 & 0,0157784 & 2,77 & 0,006 & 0,0215169 & 0,0886655 \\
\hline$\lambda$ & 3,213429 & 0,0465122 & 69,09 & 0,000 & 3,122266 & 3,304591 \\
\hline
\end{tabular}

Примечание. Составлено автором. Число наблюдений - 170, число групп - 17, критерий Вальда $\chi^{2}=173429,15$, значение симулятивной логарифмической функции правдоподобия $\mathrm{SML}=117,5746$. 
Коэффициенты корреляции между показателями функционирования СЭС, рассчитанные по панельным данным

\begin{tabular}{|l|c|c|c|c|c|c|}
\hline Показатель & $\Xi$ & JMLS (h) & BC(h) & BC(e) & JMLS (t) & BC(t) \\
\hline$\xi$ & 1 & & & & & \\
\hline JMLS(h) & 0,179198 & & & & & \\
& $(2,347)$ & & & & & \\
& {$[0,010]$} & 1 & & & & \\
BC(h) & 0,201902 & 0,538225 & & & & \\
& $(2,656)$ & $(8,228)$ & & & & \\
& {$[0,004]$} & {$[0,000]$} & 1 & & & \\
\hline BC(e) & 0,176205 & 0,528541 & 0,906774 & & & \\
& $(2,306)$ & $(8,022)$ & $(27,710)$ & & & \\
& {$[0,011]$} & {$[0,000]$} & {$[0,000]$} & 1 & & \\
\hline JMLS(t) & 0,190028 & 0,987215 & 0,608997 & 0,58664 & & \\
& $(2,494)$ & $(79,800)$ & $(9,892)$ & $(9,333)$ & & \\
& {$[0,001]$} & {$[0,000]$} & {$[0,000]$} & {$[0,000]$} & 1 & \\
\hline BC(t) & 0,224154 & 0,558938 & 0,991464 & 0,901432 & 0,628598 & \\
& $(2,963)$ & $(8,684)$ & $(97,976)$ & $(26,827)$ & $(10,413)$ & \\
& {$[0,002]$} & {$[0,000]$} & {$[0,000]$} & {$[0,000]$} & {$[0,000]$} & \\
\hline
\end{tabular}

Примечание. Составлено автором. $\xi$ - индикатор результативности; JMLS - оценка технической эффективности по: [Jondrow et al., 1982], ВC - оценка технической эффективности по: [Battese et al., 1988], h - halfnormal (полунормальное распределение), e - exponential (экспоненциальное распределение), $\mathrm{t}$ - truncated normal (усеченное распределение), (...) - t-статистика, [...] - p-value (вероятность принять гипотезу о незначимости коэффициента).

\section{Заключение}

В исследовании представлен подход к оценке результативности функционирования социально-экономических систем, включающий в себя построение частного показателя (индикатора) результативности, определяемого как отношение фактического результата к ожидаемому (нормативному, нормальному, эталонному) значению. Ожидаемое значение вычисляется по модели связи результативного признака с существенными (выделенными как значимые в процессе построения модели) факторами, которые можно разделить на факторы состояния и воздействия. Отличием от известных методов оценки функционирования СЭС является то, что предложенный метод конструирования индикатора позволяет сравнивать функционирование СЭС, имеющих различные ресурсные возможности, а также условия, которые в явном виде не могут быть отражены в модели. При этом его представление более наглядно по сравнению с показателями технической эффективности.

Для обоснования возможности использования показателя результативности в ка- честве индикатора оценки функционирования СЭС наряду с другими индикаторами был проведен анализ его статистической зависимости с показателями технической эффективности, которые вычисляются после построения стохастической граничной производственной функции с использованием различных методологий, широко применяемых в настоящее время.

Полученные результаты (рассмотрены два варианта: для объединенных и панельных данных) свидетельствуют о статистической связи изученных индикаторов, что приводит к выводу о возможности применения показателя результативности в качестве инструмента анализа и сравнения функционирования СЭС, действующих в различных социальноэкономических условиях.

\section{ПРИМЕЧАНИЕ}

1 Автор выражает глубокую благодарность доктору экономических наук, профессору, членукорреспонденту РАН Георгию Борисовичу Клейнеру за помощь при подготовке и проведении исследования. 


\section{СПИСОК ЛИТЕРАТУРЫ}

Айвазян, С. А. Моделирование производственного потенциала на основе концепции стохастической границы. Методология, результаты статистического анализа / С. А. Айвазян, М. Ю. Афанасьев. - М. : Красанд, 2015. - 352 с.

Айвазян, С. А. Некоторые вопросы спецификации трехфакторных моделей производственного потенциала компании, учитывающих интеллектуальный капитал / С. А. Айвазян, М. Ю. Афанасьев, В. А. Руденко // Прикладная эконометрика. - 2012. - № 3 (27). С. 36-69.

Жуков, Р. А. Влияние инноваций, труда и капитала на объем валового регионального продукта как индикатора устойчивого развития: регионы Центрального федерального округа и Тульская область / Р. А. Жуков, В. А. Поляков, М. В. Васина // Научные исследования и разработки. Экономика. - 2018. - Т. 6, № 5. - C. 4-9. - DOI: 10.12737/article_5bcf0ee258e203.74618577.

Жуков, Р. А. Социо-эколого-экономические системы: теория и практика : монография / Р. А. Жуков.М. : ИНФРА-М, 2019. - 186 c. - DOI: 10.12737/ monography 5b7516626665a8.43347695.

Котарбинский, Т. Трактат о хорошей работе / Т. Котарбинский. - М. : Экономика, 1975. - 249 с.

Малахов, Д. И. Методы оценки показателя эффективности в моделях стохастической производственной границы / Д. И. Малахов, Н. П. Пильник // Экономический журнал ВШЭ. - 2013. № 4. - C. 692-718.

Aigner, D. Formulation and Estimation of Stochastic Frontier Production Function Models / D. Aigner, C. A. K. Lovell, P. Schmidt // Journal of Econometrics. - 1977. - Vol. 6, № 1. - P. 21-37.

Battese, G. E. Prediction of firm-level technical efficiencies with a generalized frontier production function and panel data / G. E. Battes, T. J. Coelli // Journal of Econometrics. - 1988. - Vol. 38. P. 387-399.

Broeck van den, J. Stochastic frontier models: a Bayesian perspective / J. Broeck van den, G. Koop, J. Osiewalski, M. F. J. Steel // Journal of Econometrics. - 1994. - Vol. 61, № 2. - P. 273-303.

Charnes, A. Measuring the efficiency of decision making units / A. Charnes, W. W. Cooper, E. Rhodes // European Journal of Operational Research. - 1978. - № 2 (6). - P. 429-444.

Cobb, C. W. A Theory of Production / C. W. Cobb, P. H. Douglas // American Economic Review. 1928. - № 18. - P. 139-165.

Debreu, G. The Coefficient of Resource Utilization / G. Debreu // Journal of the Econometric Society. - 1951. - Vol. 19, № 3. - P. 273-292.
Farrel, M. G. The measurement of productive efficiency / M. G. Farrel // Journal of The Royal Statistical Society. - 1957. - Vol. 120, № 3. - P. 253-281. DOI: $10.2307 / 2343100$.

Greene, W. Reconsidering heterogeneity in panel data estimators of the stochastic frontier model / W. Greene // Journal of Econometrics. - 2005. Vol. 126. - P. 269-303.

Jondrow, J. On the Estimation of Technical Inefficiency in Stochastic Frontier Production Function Model / J. Jondrow, C. A. K. Lovell, I. S. Materov, P. Schmidt // Journal of Econometrics. - 1982. Vol. 19. - P. 233-239.

Kim, Y. A review and empirical comparison of bayesian and classical approaches to inference on efficiency levels in stochastic frontier models with panel data/Y. Kim, P. Schmidt // Journal of Productivity Analysis. - 2000. - Vol. 14, № 2. P. 91-118.

Leibenstein, H. “Allocative efficiency" vs. "X-efficiency" / H. Leibenstein // American Economic Review. 1966. - Vol. 56, № 3. - P. 392-415.

Makieta K. Bayesian Stochastic Frontier Analysis with MATLAB / K. Makieta. - Electronic text data. - Mode of access: https://www. researchgate.net/publication/319244559 (date of access: 17.02.2019). - Title from screen.

Wooldridge, J. M. Econometric Analysis of Cross Section and Panel Data / J. M. Wooldridge. MIT Press, 2002. - 735 p.

\section{REFERENCES}

Ayvazyan S.A., Afanasyev M.Yu. Modelirovanie proizvodstvennogo potentsiala na osnove kontseptsii stokhasticheskoy granitsy. Metodologiya, rezultaty statisticheskogo analiza [Development of Production Capacity on the Basis of the Stochastic Frontier. Concept: Methodology and Results of Empirical Analysis]. Moscow, Krasand Publ., 2015. 352 p.

Ayvazyan S.A., Afanasyev M.Yu., Rudenko V.A. Nekotorye voprosy spetsifikatsii trekhfaktornykh modeley proizvodstvennogo potentsiala kompanii, uchityvayushchikh intellektualnyy kapital [Some Issues on the Specification of Three-Factor Models of the Company's Production Potential Taking into Account Intellectual Capital]. Prikladnaya ekonometrika [Applied Econometrics], 2012, no. 3 (27), pp. 36-69.

Zhukov R.A., Polyakov V.A., Vasina M.V. Vliyanie innovatsiy, truda i kapitala na obyem valovogo regionalnogo produkta kak indikatora ustoychivogo razvitiya: regiony Tsentralnogo federalnogo okruga i Tulskaya oblast [The 
Impact of Innovation, Labour and Capital in Gross Regional Product As an Indicator of Sustainable Development: Regions of the Central Federal District and Tula Region]. Nauchnye issledovaniya i razrabotki. Ekonomika [Scientific Research and Development. Economics], 2018, vol. 6, no. 5, pp. 4-9. DOI: 10.12737/article_5bcf0ee258e203.74618577.

Zhukov R.A. Sotsio-ekologo-ekonomicheskie sistemy: teoriya i praktika: monografiya [Socio-Ecological and Economic Systems: Theory and Practice. Monograph]. Moscow, INFRA-M Publ., 2019. 186 p. DOI: 10.12737/ monography_5b7516626665a8.43347695.

Kotarbinskiy T. Traktat o khoroshey rabote [Treatise on Good Work]. Moscow, Ekonomika Publ., 1975. $249 \mathrm{p}$.

Malakhov D.I., Pilnik N.P. Metody otsenki pokazatelya effektivnosti v modelyakh stokhasticheskoy proizvodstvennoy granitsy [Methods of Estimating of the Efficiency in Stochastic Frontier Models]. Ekonomicheskiy zhurnal VShE [HSE Economic Journal], 2013, no. 4, pp. 692-718.

Aigner D., Lovell C.A.K., Schmidt P. Formulation and Estimation of Stochastic Frontier Production Function Models. Journal of Econometrics, 1977, vol. 6, no. 1, pp. 21-37.

Battese G.E., Coelli T.J. Prediction of Firm-Level Technical Efficiencies with a Generalized Frontier Production Function and Panel Data. Journal of Econometrics, 1988, vol. 38, pp. 387-399.

Broeck van den J., Koop G., Osiewalski J., Steel M. F. J. Stochastic Frontier Models: Bayesian Perspective. Journal of Econometrics, 1994, vol. 61 , no. 2 , pp. 273-303.
Charnes A., Cooper W.W., Rhodes E. Measuring the Efficiency of Decision Making Units. European Journal of Operational Research, 1978, no. 2 (6), pp. 429-444.

Cobb C.W., Douglas P.H. A Theory of Production. American Economic Review, 1928, no. 18, pp. 139-165.

Debreu G. The Coefficient of Resource Utilization. Journal of the Econometric Society, 1951, vol. 19, no. 3, pp. 273-292.

Farrel M.G. The Measurement of Productive Efficiency. Journal of The Royal Statistical Society, 1957, vol. 120, no. 3, pp. 253-281. DOI: 10.2307/2343100.

Greene W. Reconsidering Heterogeneity in Panel Data Estimators of the Stochastic Frontier Model. Journal of Econometrics, 2005, vol. 126, pp. 269-303.

Jondrow J., Lovell C.A.K., Materov I.S., Schmidt P. On the Estimation of Technical Inefficiency in Stochastic Frontier Production Function Model. Journal of Econometrics, 1982, vol. 19, pp. 233-239.

Kim Y., Schmidt P. A Review and Empirical Comparison of Bayesian and Classical Approaches to Inference on Efficiency Levels in Stochastic Frontier Models with Panel Data. Journal of Productivity Analysis, 2000, vol. 14, no. 2, pp. 91-118.

Leibenstein H. "Allocative Efficiency" vs. "X-Efficiency". American Economic Review, 1966, vol. 56, no. 3, pp. 392-415.

Makieta K. Bayesian Stochastic Frontier Analysis with MATLAB. URL: https://www.researchgate.net/ publication/319244559 (accessed 17 February 2019).

Wooldridge J.M. Econometric Analysis of Cross Section and Panel Data. MIT Press, 2002. 735 p.

\section{Information about the Author}

Roman A. Zhukov, Candidate of Sciences (Physics and Mathematics), Associate Professor, Department of Mathematics and Informatics, Financial University under the Government of the Russian Federation (Tula Branch), Oruzheynaya St., 1a, 300012 Tula, Russian Federation, pluszh@mail.ru, https:// orcid.org/0000-0002-2280-307X

\section{Информация об авторе}

Роман Александрович Жуков, кандидат физико-математических наук, доцент кафедры математики и информатики, Финансовый университет при Правительстве РФ (Тульский филиал), ул. Оружейная, 1a, 300012 г. Тула, Российская Федерация, pluszh@mail.ru, https://orcid.org/ 0000-0002-2280-307X 